\title{
L-Arginine Enhances Resistance against Oxidative Stress and Heat Stress in Caenorhabditis elegans
}

\author{
Heran Ma ${ }^{1}$, Yudan Ma ${ }^{2}$, Zhixian Zhang ${ }^{1}$, Ziyuan Zhao ${ }^{1}$, Ran Lin ${ }^{1}$, Jinming Zhu ${ }^{3}$, Yi Guo ${ }^{1, *}$ \\ and $\operatorname{Li~Xu}{ }^{1, *}$ \\ 1 Key laboratory for Molecular Enzymology and Engineering, The Ministry of Education, \\ National Engineering Laboratory for AIDS Vaccine, School of Life Sciences, Jilin University, \\ Changchun 130012, China; mahr13@mails.jlu.edu.cn (H.M.); zhangzhixian_99@126.com (Z.Z.); \\ 18743025536@163.com (Z.Z.); avalinran@outlook.com (R.L.) \\ 2 Sports Science Research Institute of Jilin Province, Changchun 130022, China; yudanma@sina.cn \\ 3 The China-Japan Union Hospital of Jilin University, Changchun 130033, China; zhujinmingWT@163.com \\ * Correspondence: guoyi@jlu.edu.cn (Y.G.); xuli@jlu.edu.cn (L.X.); \\ Tel.: +86-431-85155226 (Y.G.); +86-431-85155246 (L.X.)
}

Academic Editors: Matthew Lee Smith and Marcia G. Ory

Received: 4 May 2016; Accepted: 20 September 2016; Published: 29 September 2016

\begin{abstract}
The antioxidant properties of L-arginine (L-Arg) in vivo, and its effect on enhancing resistance to oxidative stress and heat stress in Caenorhabditis elegans were investigated. C. elegans, a worm model popularly used in molecular and developmental biology, was used in the present study. Here, we report that L-Arg, at a concentration of $1 \mathrm{mM}$, prolonged C. elegans life by $26.98 \%$ and $37.02 \%$ under oxidative and heat stress, respectively. Further experiments indicated that the longevity-extending effects of L-Arg may be exerted by its free radical scavenging capacity and the upregulation of aging-associated gene expression in worms. This work is important in the context of numerous recent studies that concluded that environment stresses are associated with an increased population death rate.
\end{abstract}

Keywords: C. elegans; L-Arg; antiaging; antioxidant; free radical

\section{Introduction}

It is well known that the overproduction of reactive oxygen species (ROS) leads to oxidative stress. Since oxidative stress may cause cellular damage, it has been linked to several chronic diseases, including cancer, diabetes, Alzheimer's disease, and others [1]. These diseases are characterized by an imbalance between the antioxidant enzyme system and ROS production, leading to further damage [2]. A body of evidence revealed that cellular metabolic waste products may play a major role in oxidative tissue damage [3,4]. More recently, a growing interest has been focused on the natural antioxidant compounds present in plants, animals, and even microbes [5-7]. As a semi-essential amino acid, L-Arg is readily available from a variety of food sources and has many important functions in living organisms as well as in various diseases [8-10]. Since L-Arg is used as a biosynthetic precursor of nitric oxide (NO) in mammals, it has been extensively studied and used to develop a variety of bioactive compounds. Although chronic provision of exogenous L-Arg is thought to impair NO production, known as the "arginine paradox", acute L-Arg supplementation improves NO production and is recommended under special circumstances [11]. L-Arg concentration directly determines the activity of arginase, the production of NO, urea, citrulline and ornithine [12]. Moreover, L-Arg is also implicated in insulin resistance [13,14], heat stress [15], oxidative stress [16], and many other environmental stresses. These physiological functions of L-Arg may be attributed to its regulating effects on some aging or resistance associated genes. Liang et al. found dietary L-Arg could activate the target of rapamycin 
(TOR) pathway and promote TOR-related protein synthesis in Megalobrama amblycephala [17]. Zhu et al. reported that L-Arg supplementation could alleviate hepatic heat stress in Pekin duck [15]. The main property of L-Arg is to generate NO in vivo. Can all of these physiological effects be attributed to NO?

In this study, we used Caenorhabditis elegans, as a model organism to investigate whether L-Arg could enhance resistance to heat and oxidative stresses. It has been reported that NO can increase longevity, enhance stress resistance or produce other beneficial consequences [18-20]. Although L-Arg can be used to synthesize NO by nitric oxide synthase (NOS) for most organisms, ranging from yeasts to mammals, C. elegans lacks NOS and therefore cannot synthesize NO [21]. A question arises: is C. elegans able to enhance environmental stress resistance by directly using L-Arg, even in the absence of NO? To test this hypothesis, the worms were fed with live OP50 Escherichia coli as neither C. elegans nor E. coli can produce NO. These worms lived longer and were characterized by an increased activity of ROS-detoxifying enzymes and a lower production of ROS. Several factors led to longevity or increased stress resistance: some aging-associated genes or antioxidant genes were upregulated [22]. These findings are consistent with the theory that free radicals regulate the aging process.

In this article, the antiaging effects of L-Arg were investigated in vivo. L-Arg exerts a significant antioxidant activity and improves resistance to environment stresses in C. elegans. Our results suggest that the mechanism by which L-Arg provides strong protection against stress may be based on scavenging ROS and upregulating the expression of longevity genes, such as $h s p-16.2$, sod-3, daf-2, and daf-16. Since much evidence shows that environment stresses have long been associated with population death rate, this work is meaningful to further aging study.

\section{Materials and Methods}

\subsection{Reagents}

$\mathrm{H}_{2}$ DCF-DA (2',7'-dichlorodihydro fluoresceindiacetate), a fluorescent probe, was used to determine the ROS levels in C. elegans. Juglone (5-hydroxy-1,4-naphthoquinone) was used to promote ROS generation and cause oxidative stress in worms. TRIzol reagent (Invitrogen, Carlsbad, CA, USA) was used for RNA extraction. Unless otherwise stated, all reagents used were of analytical grade and obtained from Sigma Chemical Co. (Saint Louis, MO, USA).

\subsection{Worm Strains and Maintenance}

C. elegans was grown in a standard nematode growth medium (NGM) at $20^{\circ} \mathrm{C}$ and fed with live OP50 Escherichia coli. Synchronization: Egg-laying worms were transferred to a new NGM plate and incubated for $3 \mathrm{~h}$, following which, worms were removed and the eggs were left on the plate.

The wild-type strain Bristol N2 and the transgenic strain CF1553 (muIs84) were obtained from the Caenorhabditis Genetics Center (CGC, Sao Paulo, MN, USA). The SOD-3::GFP-linked reporter present in CF1553 was used to visualize SOD-3 expression. To visualize the expression of HSP-16.2, the transgenic strain CL2070 (dvIs70)—a gift from the University of Maryland-was employed as it contains a HSP-16.2::GFP-linked reporter.

\subsection{Stress Resistance Assay}

After reaching adulthood, worms were treated with or without L-Arg $(1 \mathrm{mM})$, and a sub-population was transferred into a constant temperature incubator at $35^{\circ} \mathrm{C}$. Worm death was recorded and is presented as death/h $[23,24]$. To detect the expression of HSP-16.2, worms that had just reached adulthood were heat-shocked $\left(35^{\circ} \mathrm{C}\right.$ for $1 \mathrm{~h}$ ), and allowed to recover for $24 \mathrm{~h}$ before fluorescence microscopy analysis [25].

For the oxidative stress assays, worms that had just reached adulthood were treated with or without L-Arg $(1 \mathrm{mM})$ for $48 \mathrm{~h}$ and subsequently transferred to juglone $(500 \mu \mathrm{M})$-containing plates. Worm death was recorded and is presented as death/h. To detect the expression of SOD-3, 2-day-old 
worms were treated with or without L-Arg $(1 \mathrm{mM})$ and subsequently treated with $300 \mu \mathrm{M}$ juglone. After a $24 \mathrm{~h}$ recovery period, worms were observed under a fluorescence microscope.

All the aforementioned tests were repeated three times as independent parallel experiments and were conducted in a double-blind manner. All the experiments were performed with 200 worms; forty animals per group were collected from a plate well.

\subsection{Intracellular ROS Measurement in C. elegans}

$\mathrm{H}_{2}$ DCF-DA was used as a fluorescence molecular probe to detect the concentration of intracellular ROS in worms. To increase the oxidative stress in vivo, young adult worms were pretreated with juglone $(300 \mu \mathrm{M})$ for $1 \mathrm{~h}$ and subsequently cultured with or without L-Arg $(1 \mathrm{mM})$ for $48 \mathrm{~h}$. Afterward, worms were collected by washing the plates with M9 buffer three times to remove the remaining bacteria, and then centrifuged at low speed (4000 rpm for $30 \mathrm{~s}$ ). Worms were then divided into a 96-well plate containing an M9 buffer. $\mathrm{H}_{2}$ DCF-DA can penetrate into cells and bind intracellular ROS, thereby emitting fluoresce. The sample was excited at $485 \mathrm{~nm}$ and a microplate reader (Thermo Fisher, Waltham, MA, USA) was used collect the emission at $538 \mathrm{~nm}$. Fluorescence data were collected every $30 \mathrm{~min}$ [26].

\subsection{Fluorescence Quantification and Visualization}

The regulatory effects of L-Arg on SOD-3 and HSP-16.2 expression were investigated. CF1553 and CL2070 worms, which just reached adulthood, were tread with or without $1 \mathrm{mM}$ L-Arg. Forty worms per well (96-well plate) were suspended in a $100 \mu \mathrm{L}$ M9 buffer and a plate reader (Thermo Fisher) was used to detect fluorescence. Five parallel experiments were performed for each group. Worms were treated as described above, were narcotized with $10 \mathrm{mM}$ levamisole and fixed on a glass slide containing agarose (3\%) for observation under a fluorescence microscope. The green fluorescence intensity corresponded to the expression of SOD-3 or HSP-16.2.

\subsection{Quantitative Real-Time PCR}

After $48 \mathrm{~h}$ of incubation with or without L-Arg ( $1 \mathrm{mM})$, 4-day-old worms were used for quantitative RT-PCR analysis. A TRIzol reagent was used for total RNA extraction. The primers used in RT-PCR are listed below: ama-1, 5'-CTGACCCAAAGAACACGGTGA- $3^{\prime}$ and $5^{\prime}$-TCCAATTCGATCCGAAGAAGC-3'; sod-3, 5'-AGCATCATGCCACCTACGTGA-3' and $5^{\prime}$-CACCACCATTGAATTTCAGCG-3'; $h s p-16.2, \quad 5$ '-CGTCGAAGAGAATACTGCTGAA-3'

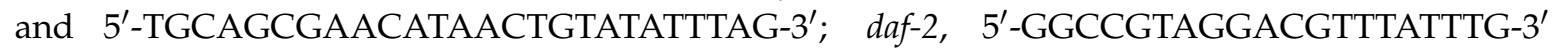
and $5^{\prime}$-TTCCACAGTGAAGAAGCCTGG-3'; daf-16, $5^{\prime}$-TTTCCGTCCCCGAACTCAA-3', and 5'-ATTCGCCAACCCATGATGG-3'.

Ama-1 was used as a reference gene; a Rotor-Gene 6000 RT-PCR detection system was used. RT-PCR data were analyzed using the comparative $2^{-\Delta \Delta C t}$ method [27].

\subsection{Statistical Analyses}

The results were presented as mean \pm standard deviation (S.D.) One-way ANOVA followed by the Tukey HSD test was performed to compare each group. Statistical significance was set at values of $p<0.05$. All statistical analyses were conducted with Origin 8.0 software (OriginLab Co., Northampton, MA, USA).

\section{Results and Discussion}

\subsection{L-Arg Protect Worms from Environment Stresses}

Under stress conditions, worms activate their defense mechanisms before developing resistance. However, if worms cannot overcome the stress themselves, exogenous supplements may be useful. In oxidative stress test experiments, 2-days-old adult worms were treated with increasing L-Arg 
concentrations $(0,0.1,1,10,50 \mathrm{mM})$ for 2 days before being transferred to a plate containing $500 \mu \mathrm{M}$ juglone. As a pro-oxidant, juglone induces oxidative damage by converting oxygen to superoxide anion, thereby increasing intracellular ROS production [28,29]. We found that $1 \mathrm{mM} \mathrm{L}-A$ rg improved mean survival by $37.02 \%$ compared to worms under oxidative stress that did not receive amino acid supplementation (Figure 1A). Next, we examined the ability of L-Arg to protect worms from heat-stress. We found that $1 \mathrm{mM}$ L-Arg $(1 \mathrm{mM})$ improved the survival time by $26.98 \%$, compared to control (Figure 1B). These results showed that L-Arg could significantly increase the mean survival time under both oxidative and heat stress. Therefore, we can hypothesize that L-Arg mixed with OP50 may be intestinally absorbed by worms and further improve resistance against environment stresses. As our result showed, L-Arg had a significant protective effect under both oxidative stress and heat stress, since the survival rate of worms corresponded with the protective ability of L-Arg on worms under environment stresses. However, chronic supplementation with L-Arg does not extend the lifespan of worms under normal conditions (Figure S1). Moreover, in order to determine whether this effect was specific to L-Arg, D-Arg was used in the same experiments and did not show any extending effects under either oxidative stress or heat stress (Figure S2A,B).
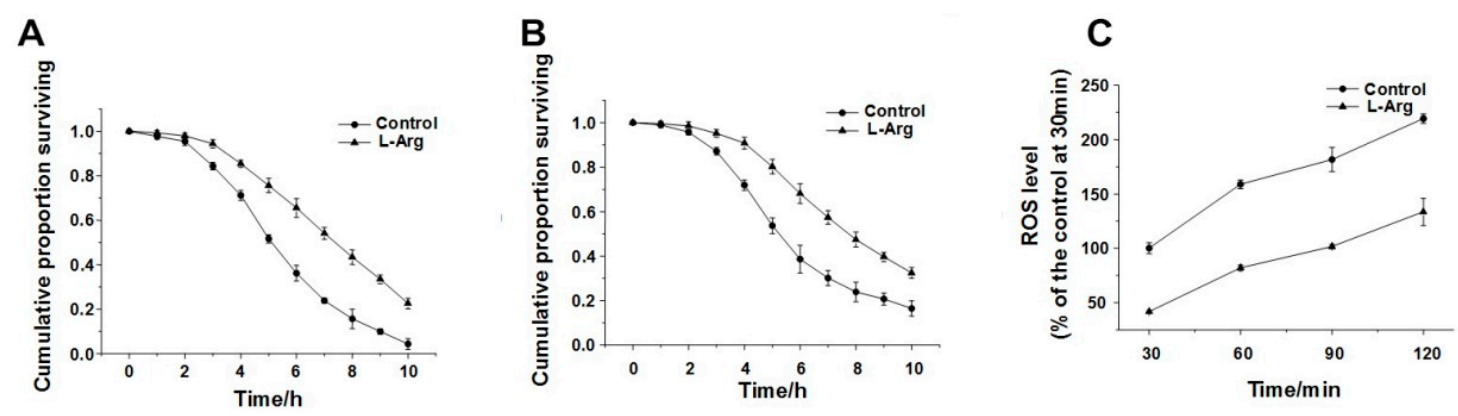

Figure 1. L-Arg enhances the resistance of C. elegans to stress conditions. (A) Protective effects of L-Arg (1 mM) on N2 wild-type C. elegans under oxidative stress; (B) Protective effects of L-Arg (1 mM) on N2 wild-type C. elegans under heat stress; (C) L-Arg at a concentration of $1 \mathrm{mM}$ decreased ROS accumulation in C. elegans in response to juglone-induced oxidative stress, over time. All experiments were conducted in triplicate and were conducted in a double-blind manner.

\subsection{L-Arg Reduced the Level of Intracellular ROS}

According to the free radical theory, excessive free radical accumulation harms different organisms. In the case of aerobic organisms, cell respiration is usually considered one of the chief culprits of oxidative damage as oxygen is used as an electron acceptor, hence promoting the formation and accumulation of ROS. Meanwhile, the newly generated free radicals, which mainly consist of superoxide anions, hydrogen peroxide and hydroxyl radicals, are immediately eliminated by antioxidant enzyme systems [3]. Therefore, we further analyzed whether the longevity effect of L-Arg under environmental stress was associated with its ROS scavenging ability. The usually high levels of ROS generated as a stress response were significantly decreased in worms treated with L-Arg compared to the control. We suggest that L-Arg may act as an antioxidant by scavenging free radicals accumulated in C. elegans and ultimately improving resistance to environment stress (Figure 1C).

\subsection{L-Arg Upregulates SOD-3::GFP in CF1553 Worms}

The intracellular ROS were scavenged by L-Arg and antioxidant enzymes such as SOD-3. However, L-Arg might not only scavenge ROS, but also positively regulate the expression of antioxidant enzymes. Here, we quantitatively determine the expression of SOD-3 with a microplate reader and a fluorescence microscope. To visualize the expression of SOD-3, the transgenic worms CF1553, expressing a SOD-3::GFP reporter, were employed. L-Arg-treated worms displayed a higher fluorescence intensity than the control group (Figure 2A). When we quantified these data, we found 
that the expression of SOD-3::GFP was significantly upregulated by $281.12 \%$ (Figure 2B). These results suggest that L-Arg may also activate antioxidant-related signaling pathways and the consequent upregulation of SOD-3 may contribute to the increased resistance to oxidative stress. Under normal conditions, L-Arg does not show a significant upregulating effect on SOD-3 (Figure S3B,D).

A

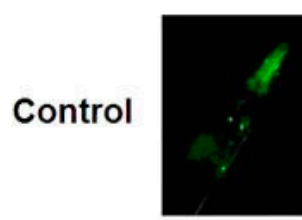

L-Arg

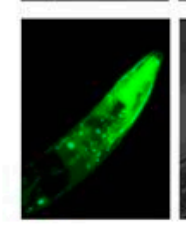

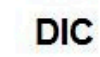
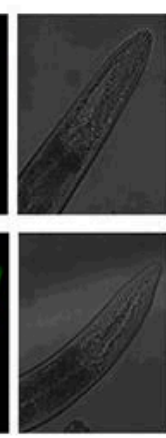
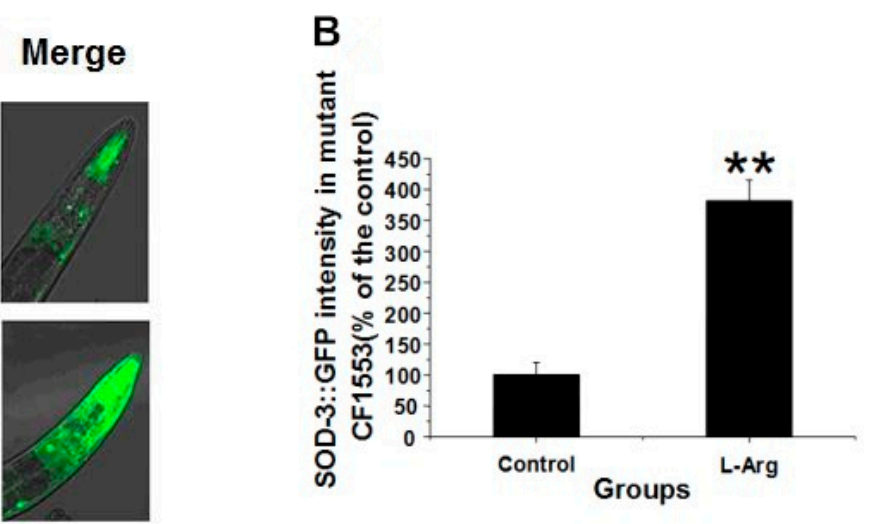

Figure 2. L-Arg upregulates SOD-3::GFP in CF1553 worms. (A) Images of SOD-3::GFP expression in L-Arg-treated and control worms; (B) Quantitative fluorescence analysis in transgenic CF1553 worms. The data are presented as the mean \pm SE of four individual experiments, with 40 worms in each group $\left({ }^{* *} p<0.01\right)$.

\subsection{L-Arg Upregulates HSP-16.2::GFP in CL2070 Worms}

HSP-16.2 is generally accepted as an indicator of longevity in C. elegans, and high HSP-16.2 expression is usually linked to a longer lifespan $[25,30]$. We therefore tested whether L-Arg might increase HSP-16.2 expression in CL2070 worms, expressing the recombinant HSP-16.2::GFP reporter gene. Worms underwent a heat-shock before a 24-h recovery period prior to fluorescence measurement. Compared to the control group, L-Arg treated worms displayed a higher degree of fluorescence (Figure 3A). When we quantified data, we found that the fluorescence intensity increased by $42.28 \%$ compared with the control (Figure 3B). Since the fluorescence intensity corresponds to the expression of HSP-16.2, we conclude that the L-Arg might prolong the meansurvival time under heat-stress by upregulating HSP-16.2. Under normal conditions, L-Arg did not show a significant upregulating effect on HSP-16.2 (Figure S3A,C).

A

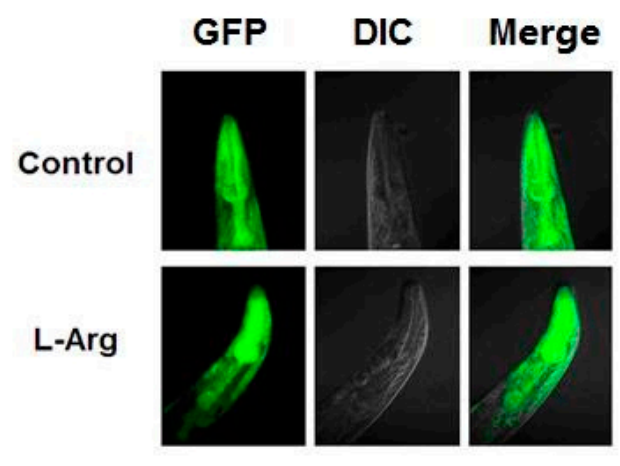

B

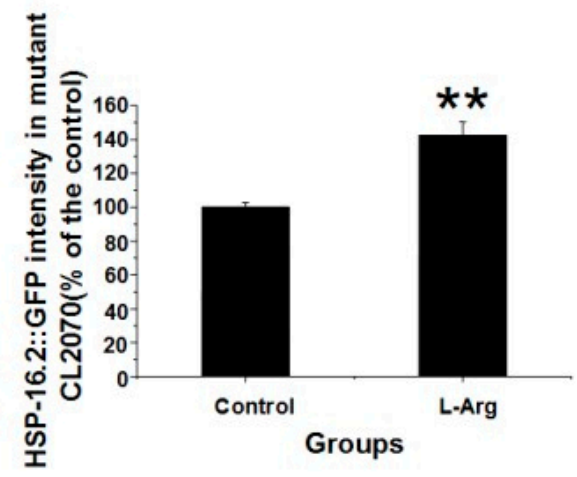

Figure 3. L-Arg upregulates HSP-16.2::GFP expression in CL2070 worms. (A) Images of HSP-16.2::GFP expression in control and L-Arg-treated worms; (B) Quantitative fluorescence analysis in transgenic CL2070 worms. Data are presented as the mean \pm SE of four individual experiments, with 40 worms in each group $(* * p<0.01)$. 


\subsection{L-Arg Regulates the Insulin/IGF Signaling Pathway in C. elegans}

To further investigate the relationship between the longevity and the regulatory effect of L-Arg on aging-associated signaling pathways, we performed RT-PCR analysis. According to the results, L-Arg appeared to increase the expression of $d a f-16$ and subsequently upregulates the daf- 16 downstream genes $h s p-16.2$ and sod-3; while reducing daf-2 expression (Figure 4A). $h s p-16.2$ and sod-3 upregulation is directly responsible for the longevity effect of L-Arg under heat and oxidative stress [31,32]. For most species, from yeasts to humans, the mechanisms of aging are highly conserved and linked to the insulin/IGF signaling (IIS) pathway [32,33]. In nematodes, the daf-16 gene encodes the forehead transcription factor DAF-16 which is downstream of the IIS pathway and plays a universal role in nematode aging and stress resistance [34-36]. In order to perform its regulatory function in the nucleus, DAF-16 needs to inhibit the IIS pathway since IIS activation will lead to DAF-16 phosphorylation and consequent retention in the cytoplasm [36-40]. Once DAF-16 translocates into the nucleus, it binds to various promoters to regulate the transcription of target genes, such as MnSOD (Mn-superoxide dismutase), encoded by the sod-3 gene in C. elegans, which would enhance resistance against oxidative stress [41,42]. Apart from sod-3, many other downstream effectors exert a regulatory effect on lifespan or stress resistance [43]. Published studies have reported that the overexpression of $h s p-16.2$ also plays a role in C. elegans longevity $[25,44,45]$. Therefore, the C. elegans insulin/IGF receptor DAF-2 negatively regulates metabolic activity and accelerates aging. Our results showed that L-Arg significantly downregulates daf-2 and upregulates daf-16. The mechanism of L-Arg-induced longevity may be as follows: DAF-2 downregulation in the cellular membrane will increase DAF-16 expression while, at the same time, reduce the IIS pathway. The latter will result in the upregulation of genes such as $h s p-16.2$ and sod-3 (Figure 4B). These findings may partially explain how L-Arg enhances resistance to environmental stress, while increasing the survival rate.

A

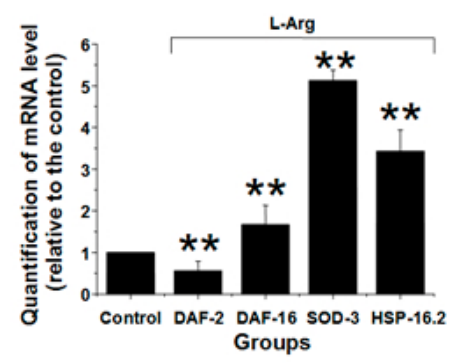

B

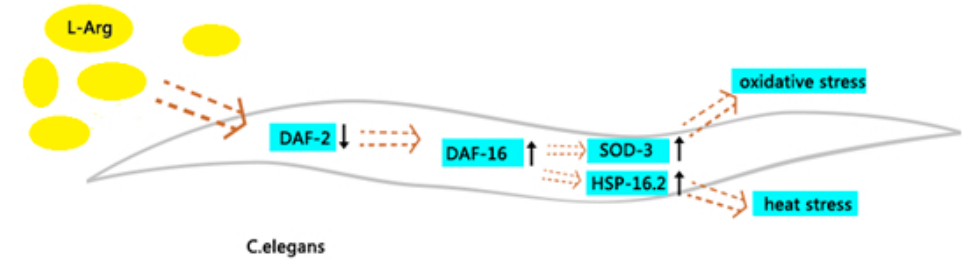

Figure 4. The regulatory effects of L-Arg on ageing-associated genes in C. elegans. (A) Worms were treated with or without $1 \mathrm{mM}$ L-Arg. RT-PCR was performed to quantify the expression of the ageing-associated genes in $C$. elegans. Data are presented as mean $\pm \mathrm{SE}(* * p<0.01)$; (B) Proposed mechanism of action: L-Arg regulates IIS in C. elegans by downregulating the expression of DAF-2 receptor and subsequently triggering the overexpression of daf-16 and its downstream target genes, and ultimately enhancing the resistance against the environment stresses.

\section{Conclusions}

In conclusion, we found that L-Arg extends the lifespan of C. elegans under both oxidative and heat stress. L-Arg not only has free radical scavenging ability but also regulates the IIS pathway and may be co-administered for improved stress-resistance in nematodes. L-Arg exists in several health products and the above findings add to a growing body of evidence that show the significance of the insulin/IGF signaling pathway as an ageing mechanism in nematodes and potentially in humans.

Supplementary Materials: The following are available online at www.mdpi.com/1660-4601/13/10/969/s1. Figure S1: Lifespan experiment showing the effect of L-Arg on wild type C. elegans N2; Figure S2: D-Arg does not enhance the resistance of $C$. elegans under stress conditions. (A) D-Arg ( $1 \mathrm{mM})$ does not affect the longevity 
of wild type C. elegans N2 under oxidative stress; (B) D-Arg (1 mM) does not affect the longevity of wild type C. elegans N2 under heat stress; Figure S3: The regulatory effect of L-Arg on HSP-16.2::GFP and SOD-3::GFP in mutant worms. (A) Images of HSP-16.2::GFP expression in L-Arg-treated and control worms; (B) Images of SOD-3::GFP expression in L-Arg-treated and control worms; (C) Quantitative fluorescence analysis in transgenic CL2070 worms; (D) Quantitative fluorescence analysis in transgenic CF1553 worms. The data are presented as the mean \pm SE.

Acknowledgments: This work is supported by the National Natural Science Foundation of China (No. 81571791, 31571017, 81271697), the Specialized Research Fund for the Doctoral Program of Higher Education of China (No. 20120061110021).

Author Contributions: Yi Guo and Li Xu conceived and designed the experiments. Heran Ma and Yudan Ma performed the experiments and contributed to the writing of the manuscript. Heran Ma, Yudan Ma, Zhixian Zhang, Ziyuan Zhao and Ran Lin analyzed data. Jinming Zhu, Yi Guo and Li Xu contributed reagents/materials/analysis tools.

Conflicts of Interest: The authors declare that they have no conflict of interest.

\section{Abbreviations}

The following abbreviations are used in this manuscript:

$\begin{array}{ll}\text { C. elegans } & \text { Caenorhabditis elegans } \\ \text { ROS } & \text { reactive oxygen species } \\ \text { L-Arg } & \text { L-arginine } \\ \text { D-Arg } & \text { D-arginine } \\ \text { MnSOD } & \text { Mn-superoxide dismutase } \\ \text { IIS } & \text { insulin/IGF signaling }\end{array}$

\section{References}

1. An, J.H.; Vranas, K.; Lucke, M.; Inoue, H.; Hisamoto, N.; Matsumoto, K.; Blackwell, T.K. Regulation of the Caenorhabditis elegans oxidative stress defense protein SKN-1 by glycogen synthase Kinase-3. Proc. Natl. Acad. Sci. USA 2005, 102, 16275-16280. [CrossRef] [PubMed]

2. Wu, H.; Zhao, Y.; Guo, Y.; Xu, L.; Zhao, B. Significant longevity-extending effects of a tetrapeptide from maize on Caenorhabditis elegans under stress. Food Chem. 2012, 130, 254-260. [CrossRef]

3. Harman, D. Aging: A theory based on free radical and radiation chemistry. J. Gerontol. 1956, 11, $298-300$. [CrossRef] [PubMed]

4. Muller, F.L.; Lustgarten, M.S.; Jang, Y.; Richardson, A.; Van Remmen, H. Trends in oxidative aging theories. Free Radic. Biol. Med. 2007, 43, 477-503. [CrossRef] [PubMed]

5. Chen, C.; Chi, Y.J.; Zhao, M.Y.; Lv, L. Purification and identification of antioxidant peptides from egg white protein hydrolysate. Amino Acids 2012, 43, 457-466. [CrossRef] [PubMed]

6. Power, O.; Jakeman, P.; FitzGerald, R.J. Antioxidative peptides: Enzymatic production, in vitro and in vivo antioxidant activity and potential applications of milk-derived antioxidative peptides. Amino Acids 2013, 44, 797-820. [CrossRef] [PubMed]

7. Yu, J.; Hu, Y.; Xue, M.; Dun, Y.; Li, S.; Peng, N.; Liang, Y.; Zhao, S. Purification and identification of antioxidant peptides from enzymatic hydrolysate of spirulina platensis. J. Microbiol. Biotechnol. 2016, 26, 1216-1223. [PubMed]

8. Chaturvedi, R.; de Sablet, T.; Coburn, L.A.; Gobert, A.P.; Wilson, K.T. Arginine and polyamines in helicobacter pylori-induced immune dysregulation and gastric carcinogenesis. Amino Acids 2012, 42, 627-640. [PubMed]

9. Clemmensen, C.; Madsen, A.N.; Smajilovic, S.; Holst, B.; Brauner-Osborne, H. L-arginine improves multiple physiological parameters in mice exposed to diet-induced metabolic disturbances. Amino Acids 2012, 43, 1265-1275. [PubMed]

10. Popolo, A.; Adesso, S.; Pinto, A.; Autore, G.; Marzocco, S. L-arginine and its metabolites in kidney and cardiovascular disease. Amino Acids 2014, 46, 2271-2286. [CrossRef] [PubMed]

11. Dioguardi, F.S. Clinical use of amino acids as dietary supplement: Pros and cons. J. Caxhexia Sarcopenia Muscle 2011, 2, 75-80. [CrossRef] [PubMed]

12. Dioguardi, F.S. To Give or Not to Give? Lessons from the Arginine Paradox. J. Nutrigenet Nutrigenom 2011, 4 , 90-98. [CrossRef] [PubMed] 
13. Dioguardi, F.S. Wasting and the substrate-to-energy controlled pathway: A role for insulin resistance and amino acids. Am. J. Cardiol. 2004, 93, 6A-12A. [CrossRef] [PubMed]

14. Barbosa, T.D.; Poyares, L.L.; Machado, U.F.; Nunes, M.T. Chronic oral administration of arginine induces GH gene expression and insulin resistance. Life Sci. 2006, 79, 1444-1449. [CrossRef] [PubMed]

15. Zhu, W.; Jiang, W.; Wu, L.Y. Dietary L-arginine supplement alleviates hepatic heat stress and improves feed conversion ratio of Pekin ducks exposed to high environmental temperature. J. Anim. Physiol. Anim. Nutr. 2014, 98, 1124-1131. [CrossRef] [PubMed]

16. Cao, W.; Xiao, L.; Liu, G.M.; Fang, T.T.; Wu, X.J.; Jia, G.; Zhao, H.; Chen, X.L.; Wu, C.M.; Cai, J.Y.; et al. Dietary arginine and $\mathrm{N}$-carbamylglutamate supplementation enhances the antioxidant statuses of the liver and plasma against oxidative stress in rats. Food Funct. 2016, 7, 2303-2311. [CrossRef] [PubMed]

17. Liang, H.; Ren, M.; Habte-Tsion, H.-M.; Ge, X.; Xie, J.; Mi, H.; Xi, B.; Miao, L.; Liu, B.; Zhou, Q.; et al. Dietary arginine affects growth performance, plasma amino acid contents and gene expressions of the TOR signaling pathway in juvenile blunt snout bream, Megalobrama amblycephala. Aquaculture 2016, 461, 1-8. [CrossRef]

18. Goud, A.P.; Goud, P.T.; Diamond, M.P.; Abu-Soud, H.M. Nitric oxide delays oocyte aging. Biochemistry 2005, 44, 11361-11368. [CrossRef] [PubMed]

19. Kong, X.; Wang, T.; Li, W.; Tang, W.; Zhang, D.; Dong, H. Exogenous nitric oxide delays salt-induced leaf senescence in cotton (Gossypium hirsutum L.). Acta Physiol. Plant. 2016, 38. [CrossRef]

20. Gusarov, I.; Shatalin, K.; Starodubtseva, M.; Nudler, E. Endogenous nitric oxide protects bacteria against a wide spectrum of antibiotics. Science 2009, 325, 1380-1384. [CrossRef] [PubMed]

21. Liochev, S.I. Free radical paradoxes. Free Radic. Biol. Med. 2013, 65, 232-233. [CrossRef] [PubMed]

22. Honda, Y.; Honda, S. The DAF-2 gene network for longevity regulates oxidative stress resistance and Mn-superoxide dismutase gene expression in Caenorhabditis elegans. FASEB J. 1999, 13, 1385-1393. [PubMed]

23. Hansen, M.; Hsu, A.L.; Dillin, A.; Kenyon, C. New genes tied to endocrine, metabolic, and dietary regulation of lifespan from a Caenorhabditis elegans genomic RNAi screen. PLoS Genet. 2005, 1, 119-128. [CrossRef] [PubMed]

24. Lithgow, G.J.; White, T.M.; Melov, S.; Johnson, T.E. Thermotolerance and extended life-span conferred by single-gene mutations and induced by thermal stress. Proc. Natl. Acad. Sci. USA 1995, 92, 7540-7544. [CrossRef] [PubMed]

25. Rea, S.; Wu, D., Jr.; Vaupel, J.; Johnson, T. A stress-sensitive reporter predicts longevity in isogenic populations of Caenorhabditis elegans. Nat. Genet. 2005, 37, 894-898. [CrossRef] [PubMed]

26. Schulz, T.J.; Zarse, K.; Voigt, A.; Urban, N.; Birringer, M.; Ristow, M. Glucose restriction extends Caenorhabditis elegans life span by inducing mitochondrial respiration and increasing oxidative stress. Cell Metab. 2007, 6, 280-293. [CrossRef] [PubMed]

27. Livak, K.J.; Schmittgen, T.D. Analysis of relative gene expression data using real-time quantitative PCR and the 2(t)(-delta delta c) method. Methods 2001, 25, 402-408. [CrossRef] [PubMed]

28. Burmeister, C.; Luersen, K.; Heinick, A.; Hussein, A.; Domagalski, M.; Walter, R.D.; Liebau, E. Oxidative stress in Caenorhabditis elegans: Protective effects of the omega class glutathione transferase (GSTO-1). FASEB J. 2008, 22, 343-354. [CrossRef] [PubMed]

29. Kampkotter, A.; Gombitang Nkwonkam, C.; Zurawski, R.F.; Timpel, C.; Chovolou, Y.; Watjen, W.; Kahl, R. Effects of the flavonoids kaempferol and fisetin on thermotolerance, oxidative stress and foxo transcription factor daf-16 in the model organism Caenorhabditis elegans. Arch. Toxicol. 2007, 81, 849-858. [CrossRef] [PubMed]

30. Hsu, A.L.; Murphy, C.T.; Kenyon, C. Regulation of aging and age-related disease by DAF-16 and heat-shock factor. Science 2003, 300, 1142-1145. [CrossRef] [PubMed]

31. Antebi, A. Genetics of aging in Caenorhabditis elegans. PLoS Genet. 2007, 3, 1565-1571. [CrossRef] [PubMed]

32. Kenyon, C. The plasticity of aging: Insights from long-lived mutants. Cell 2005, 120, 449-460. [CrossRef] [PubMed]

33. Gems, D.; Partridge, L. Insulin/IGF signalling and ageing: Seeing the bigger picture. Curr. Opin. Genet. Dev. 2001, 11, 287-292. [CrossRef]

34. Kenyon, C.; Chang, J.; Gensch, E.; Rudner, A.; Tabtiang, R. A C. elegans mutant that lives twice as long as wild type. Nature 1993, 366, 461-464. [CrossRef] [PubMed]

35. Lin, K.; Kenyon, C. DAF-16: An HNF-3/Forkhead family member that can function to double the life-span of Caenorhabditis elegans. Science 1997, 278, 1319-1322. [CrossRef] [PubMed] 
36. Ogg, S.; Paradis, S.; Gottlieb, S.; Patterson, G.I.; Lee, L.; Tissenbaum, H.A.; Ruvkun, G. The fork head transcription factor DAF-16 transduces insulin-like metabolic and longevity signals in C. elegans. Nature 1997, 389, 994-999. [PubMed]

37. Dorman, J.B.; Albinder, B.; Shroyer, T.; Kenyon, C. The age-1 and DAF-2 genes function in a common pathway to control the lifespan of Caenorhabditis elegans. Genetics 1995, 141, 1399-1406. [PubMed]

38. Henderson, S.T.; Johnson, T.E. Daf-16 integrates developmental and environmental inputs to mediate aging in the nematode Caenorhabditis elegans. Curr. Biol. 2001, 11, 1975-1980. [CrossRef]

39. Hertweck, M.; Gobel, C.; Baumeister, R. C. elegans SGK-1 is the critical component in the Akt/PKB kinase complex to control stress response and life span. Dev. Cell 2004, 6, 577-588. [CrossRef]

40. Lin, K.; Hsin, H.; Libina, N.; Kenyon, C. Regulation of the Caenorhabditis elegans longevity protein DAF-16 by insulin/IGF-1 and germline signaling. Nat. Genet. 2001, 28, 139-145. [CrossRef] [PubMed]

41. Sun, J.T.; Folk, D.; Bradley, T.J.; Tower, J. Induced overexpression of mitochondrial Mn-superoxide dismutase extends the life span of adult drosophila melanogaster. Genetics 2002, 161, 661-672. [PubMed]

42. Sun, J.T.; Tower, J. FLP recombinase-mediated induction of $\mathrm{Cu} / \mathrm{Zn}$-superoxide dismutase transgene expression can extend the life span of adult drosophila melanogaster flies. Mol. Cell. Biol. 1999, 19, 216-228. [CrossRef] [PubMed]

43. Braeckman, B.P.; Vanfleteren, J.R. Genetic control of longevity in C. elegans. Exp. Gerontol. 2007, 42, 90-98. [CrossRef] [PubMed]

44. Walker, G.A.; Lithgow, G.J. Lifespan extension in C. elegans by a molecular chaperone dependent upon insulin-like signals. Aging Cell 2003, 2, 131-139. [CrossRef] [PubMed]

45. Wang, H.D.; Kazemi-Esfarjani, P.; Benzer, S. Multiple-stress analysis for isolation of drosophila longevity genes. Proc. Natl. Acad. Sci. USA 2004, 101, 12610-12615. [CrossRef] [PubMed]

(C) 2016 by the authors; licensee MDPI, Basel, Switzerland. This article is an open access article distributed under the terms and conditions of the Creative Commons Attribution (CC-BY) license (http://creativecommons.org/licenses/by/4.0/). 